\title{
Open spin chains and complexity in the high energy limit
}

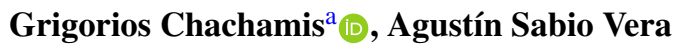 \\ Instituto de Física Teórica UAM/CSIC \& Universidad Autónoma de Madrid, Nicolás Cabrera 15, 28049 Madrid, Spain
}

Received: 23 January 2018 / Accepted: 7 June 2018 / Published online: 14 June 2018

(C) The Author(s) 2018

\begin{abstract}
In the high energy limit of scattering amplitudes in Quantum Chromodynamics and supersymmetric theories the dominant Feynman diagrams are characterized by a hidden integrability in the planar limit. A well-known example is that of Odderon exchange, which can be described as a composite state of three reggeized gluons and corresponds to a closed spin chain with periodic boundary conditions. In the $N=4$ supersymmetric Yang-Mills theory a similar spin chain arises in the multi-Regge asymptotics of the eight-point amplitude in the planar limit. We investigate the associated open spin chain in transverse momentum and rapidity variables solving the corresponding effective Feynman diagrams. We introduce the concept of complexity in the high energy effective field theory and study its emerging scaling laws.
\end{abstract}

\section{Introduction}

The high energy regime in Quantum Chromodynamics (QCD) has attracted a lot of attention for many years due to the rich structure of the theory in this limit. Novel effective degrees of freedom, reggeized quarks and gluons, arise when evaluating scattering amplitudes in the multi-Regge kinematical region (MRK). They can form composite states with different quantum numbers among which the best known are the Pomeron and the Odderon [1-3]. The (perturbative) hard Pomeron (even under charge and parity conjugation) is best described within the Balitsky-Fadin-Kuraev-Lipatov (BFKL) formalism [4-6]. It corresponds to the dominant contribution to cross sections at very large energies and, together with the Odderon (odd under charge and parity conjugation), in perturbation theory they correspond, respectively, to a composite state of two and three reggeized gluons (see Fig. 1). A large amount of effort has been expended to study the properties of the Pomeron while for the Odderon the understanding, both theoretically and phenomenologically, is more limited [7].

a e-mail: chachamis@gmail.com
The contribution to a cross section due to Odderon exchange can be calculated by solving an integral equation describing the $t$-channel exchange of three off-shell reggeized gluons, the so-called Bartels-KwiecinskiPraszalowicz (BKP) equation [8-10]. Since, in MRK, this equation is invariant under two-dimensional conformal transformations in coordinate representation, one can apply techniques developed in conformal field theories and integrable systems [11-15] to find the solution to the equation. It is remarkable the existence of a mapping to a closed spin chain (CSC) when working in impact parameter representation $[11,12]$. However, due to the different possible choices of normalization conditions for the Odderon wave function, it is difficult to know which analytic solution is relevant for a particular scattering process.

In a recent work [16], we solved the Odderon problem using an orthogonal method to those applied so far in the literature on this subject. Working in transverse momentum and rapidity space we solved exactly the equation governing the Odderon Green function. Our solution was expressed as a set of nested integrations which can be evaluated using numerical Monte Carlo techniques.

The solution to the BKP equation for three Reggeons corresponds to a six-point amplitude with off-shells gluons $f_{\omega}\left(\mathbf{p}_{1}, \mathbf{p}_{2}, \mathbf{p}_{3} ; \mathbf{p}_{4}, \mathbf{p}_{5}, \mathbf{p}_{6}\right)$ (which we write as $f_{\omega}$ $\left.\left(\mathbf{p}_{1}, \mathbf{p}_{2}, \mathbf{p}_{3}\right)\right) . \mathbf{p}_{i}$ are Euclidean two-dimensional transverse vectors where $\mathbf{p}_{i=1,2,3}$ have rapidity $Y$ and $\mathbf{p}_{i=4,5,6}$ a rapidity $0 . \omega$ is a complex variable, Mellin-conjugate of $Y$. The reggeized gluon propagators are infrared divergent and need a regulator with mass-dimension $\lambda$. With this regularization, the gluon Regge trajectory at leading order can be written in the form (with $\left.\bar{\alpha}_{s}=\alpha_{s} N_{c} / \pi\right)$

$\omega(\mathbf{p})=-\frac{\bar{\alpha}_{s}}{2} \ln \frac{\mathbf{p}^{2}}{\lambda^{2}}$.

When the square of a Lipatov's emission vertex,

$\xi\left(\mathbf{p}_{i}, \mathbf{p}_{j}, \mathbf{p}_{k}, \mathbf{k}\right)=\frac{\bar{\alpha}_{s}}{4} \frac{\theta\left(\mathbf{k}^{2}-\lambda^{2}\right)}{\pi \mathbf{k}^{2}}$ 
(1)

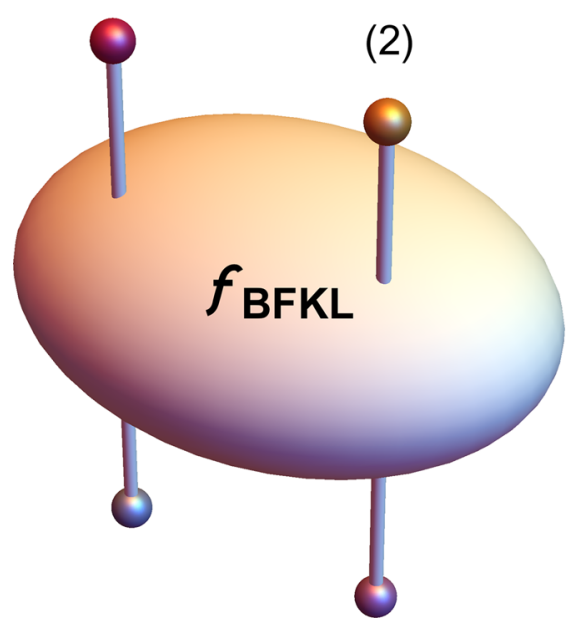

(2)

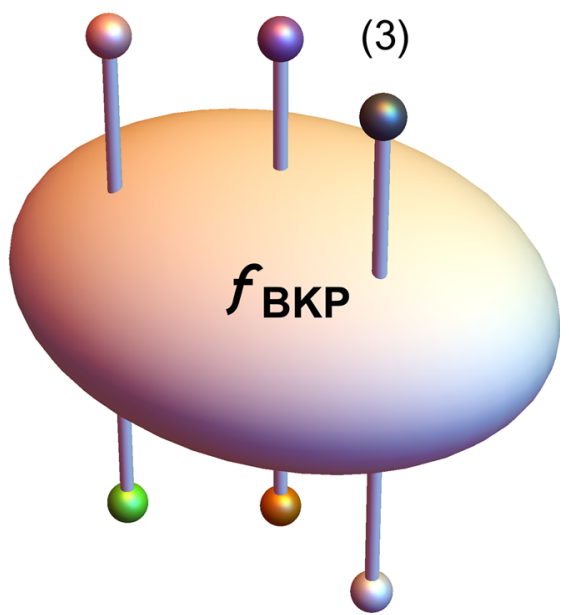

Fig. 1 Green functions describing the composite state of two (BFKL Pomeron) or three (BKP Odderon) reggeized-gluon composite states

$$
\times\left(1+\frac{\left(\mathbf{p}_{i}+\mathbf{k}\right)^{2} \mathbf{p}_{j}^{2}-\left(\mathbf{p}_{i}+\mathbf{p}_{j}\right)^{2} \mathbf{k}^{2}}{\mathbf{p}_{i}^{2}\left(\mathbf{k}-\mathbf{p}_{2}\right)^{2}}\right)
$$

is integrated over the transverse momentum of the gluon with momentum $\mathbf{k}$ it generates a further $\lambda$ dependence which cancels that of the gluon trajectory. The function $\xi$ in Eq. (2) couples two reggeized gluons with momenta $\mathbf{p}_{i}$ and $\mathbf{p}_{j}$ via a $s$-channel normal gluon with transverse momentum $\mathbf{k}$, leaving the third reggeized gluon, with momentum $\mathbf{p}_{k}$, as a spectator. This generates pairwise nearest neighbor interactions in the corresponding CSC.

The BKP equation in the Odderon case combines all these elements making use of a ternary kernel:

$$
\begin{aligned}
& \left(\omega-\omega\left(\mathbf{p}_{1}\right)-\omega\left(\mathbf{p}_{2}\right)-\omega\left(\mathbf{p}_{3}\right)\right) f_{\omega}\left(\mathbf{p}_{1}, \mathbf{p}_{2}, \mathbf{p}_{3}\right) \\
& =\delta^{(2)}\left(\mathbf{p}_{1}-\mathbf{p}_{4}\right) \delta^{(2)}\left(\mathbf{p}_{2}-\mathbf{p}_{5}\right) \delta^{(2)}\left(\mathbf{p}_{3}-\mathbf{p}_{6}\right) \\
& \quad+\int d^{2} \mathbf{k} \xi\left(\mathbf{p}_{1}, \mathbf{p}_{2}, \mathbf{p}_{3}, \mathbf{k}\right) f_{\omega}\left(\mathbf{p}_{1}+\mathbf{k}, \mathbf{p}_{2}-\mathbf{k}, \mathbf{p}_{3}\right) \\
& \quad+\int d^{2} \mathbf{k} \xi\left(\mathbf{p}_{2}, \mathbf{p}_{3}, \mathbf{p}_{1}, \mathbf{k}\right) f_{\omega}\left(\mathbf{p}_{1}, \mathbf{p}_{2}+\mathbf{k}, \mathbf{p}_{3}-\mathbf{k}\right) \\
& \quad+\int d^{2} \mathbf{k} \xi\left(\mathbf{p}_{1}, \mathbf{p}_{3}, \mathbf{p}_{2}, \mathbf{k}\right) f_{\omega}\left(\mathbf{p}_{1}+\mathbf{k}, \mathbf{p}_{2}, \mathbf{p}_{3}-\mathbf{k}\right) .
\end{aligned}
$$

The initial condition for evolution in rapidity corresponds to three reggeized-gluon propagators normalized in the form of three two-dimensional Dirac delta functions $\delta^{(2)}\left(\mathbf{p}_{1}-\mathbf{p}_{4}\right)$ $\delta^{(2)}\left(\mathbf{p}_{2}-\mathbf{p}_{5}\right) \delta^{(2)}\left(\mathbf{p}_{3}-\mathbf{p}_{6}\right)$.

In Ref. [16] we showed how to iterate the BKP ternary kernel acting on the initial condition until we reach convergence for a particular value of the relevant expansion parameter $\bar{\alpha}_{S} Y$. The gluon Green function grows with $Y$ for small values of this variable to then rapidly decrease at higher $Y$ (keeping $\bar{\alpha}_{S}$ constant). Our solution is compatible with previ- ous approaches where the Odderon intercept has been argued to be of $\mathcal{O}(1)[17,18]$ (similar results have been found within the dipole formalism [19]).

In the following section we will explain in some detail our procedure to solve an equation similar to Eq. (3) which has a representation as an integrable open spin chain (OSC) in the planar limit as shown by Lipatov in Ref. [20]. In a nutshell, we first iterate it in the $\omega$ space to then transform the result to get back to a representation with only transverse momenta and rapidity.

\section{The equation for the open spin chain}

When evaluating the eight-gluon amplitude in the $N=4$ supersymmetric theory in MRK and in certain physical regions we encounter a contribution with three Reggeized gluons exchanged in the $t$-channel. This is the most complicated contribution to the amplitude stemming from the socalled Mandelstam cuts in the associated partial wave [2023]. Since the amplitude carries the quantum numbers of a gluon in that channel, this implies that the contributing effective Feynman diagrams are planar and the gluons with momentum $\mathbf{p}_{1}$ and $\mathbf{p}_{3}$ cannot be directly connected by the function $\xi$. This is the reason why we now have an OSC. The corresponding BKP-like integral equation then reads

$$
\begin{aligned}
& \left(\omega-\omega\left(\mathbf{p}_{1}\right)-\omega\left(\mathbf{p}_{2}\right)-\omega\left(\mathbf{p}_{3}\right)\right) f_{\omega}\left(\mathbf{p}_{1}, \mathbf{p}_{2}, \mathbf{p}_{3}\right) \\
& =\delta^{(2)}\left(\mathbf{p}_{1}-\mathbf{p}_{4}\right) \delta^{(2)}\left(\mathbf{p}_{2}-\mathbf{p}_{5}\right) \delta^{(2)}\left(\mathbf{p}_{3}-\mathbf{p}_{6}\right) \\
& \quad+\int d^{2} \mathbf{k} \xi\left(\mathbf{p}_{1}, \mathbf{p}_{2}, \mathbf{p}_{3}, \mathbf{k}\right) f_{\omega}\left(\mathbf{p}_{1}+\mathbf{k}, \mathbf{p}_{2}-\mathbf{k}, \mathbf{p}_{3}\right) \\
& \quad+\int d^{2} \mathbf{k} \xi\left(\mathbf{p}_{2}, \mathbf{p}_{3}, \mathbf{p}_{1}, \mathbf{k}\right) f_{\omega}\left(\mathbf{p}_{1}, \mathbf{p}_{2}+\mathbf{k}, \mathbf{p}_{3}-\mathbf{k}\right)
\end{aligned}
$$


Following closely our approach for the CSC, we can solve this equation by iteration. With this in mind, it is convenient to use the operator notation

$$
\begin{aligned}
& \mathcal{O}(\mathbf{k}) \otimes f\left(\mathbf{p}_{1}, \mathbf{p}_{2}, \mathbf{p}_{3}\right) \\
& \quad \equiv \xi\left(\mathbf{p}_{1}, \mathbf{p}_{2}, \mathbf{p}_{3}, \mathbf{k}\right) f\left(\mathbf{p}_{1}+\mathbf{k}, \mathbf{p}_{2}-\mathbf{k}, \mathbf{p}_{3}\right) \\
& \quad+\xi\left(\mathbf{p}_{2}, \mathbf{p}_{3}, \mathbf{p}_{1}, \mathbf{k}\right) f\left(\mathbf{p}_{1}, \mathbf{p}_{2}+\mathbf{k}, \mathbf{p}_{3}-\mathbf{k}\right),
\end{aligned}
$$

to write the equation in the form

$$
\begin{aligned}
& \left(\omega-\omega\left(\mathbf{p}_{1}\right)-\omega\left(\mathbf{p}_{2}\right)-\omega\left(\mathbf{p}_{3}\right)\right) f_{\omega}\left(\mathbf{p}_{1}, \mathbf{p}_{2}, \mathbf{p}_{3}\right) \\
& =\delta^{(2)}\left(\mathbf{p}_{1}-\mathbf{p}_{4}\right) \delta^{(2)}\left(\mathbf{p}_{2}-\mathbf{p}_{5}\right) \delta^{(2)}\left(\mathbf{p}_{3}-\mathbf{p}_{6}\right) \\
& \quad+\int d^{2} \mathbf{k} \mathcal{O}(\mathbf{k}) \otimes f_{\omega}\left(\mathbf{p}_{1}, \mathbf{p}_{2}, \mathbf{p}_{3}\right) .
\end{aligned}
$$

Iterating this expression we obtain

$$
\begin{aligned}
& f_{\omega}\left(\mathbf{p}_{1}, \mathbf{p}_{2}, \mathbf{p}_{3}\right)=\frac{\left(1+\sum_{n=1}^{\infty} \prod_{i=1}^{n} \int d^{2} \mathbf{k}_{i} \mathcal{O}\left(\mathbf{k}_{i}\right) \otimes\right)}{\left(\omega-\omega\left(\mathbf{p}_{1}\right)-\omega\left(\mathbf{p}_{2}\right)-\omega\left(\mathbf{p}_{3}\right)\right)} \\
& \times \delta^{(2)}\left(\mathbf{p}_{1}-\mathbf{p}_{4}\right) \delta^{(2)}\left(\mathbf{p}_{2}-\mathbf{p}_{5}\right) \delta^{(2)}\left(\mathbf{p}_{3}-\mathbf{p}_{6}\right) .
\end{aligned}
$$

We can now trade $\omega$ for a $Y$ dependence using

$$
f\left(\mathbf{p}_{1}, \mathbf{p}_{2}, \mathbf{p}_{3}, Y\right)=\int_{a-i \infty}^{a+i \infty} \frac{d \omega}{2 \pi i} e^{\omega Y} f_{\omega}\left(\mathbf{p}_{1}, \mathbf{p}_{2}, \mathbf{p}_{3}\right)
$$

and write our solution in the form

$$
\begin{aligned}
& f_{\mathrm{BKP}}^{\text {adjoint }}\left(\mathbf{p}_{1}, \mathbf{p}_{2}, \mathbf{p}_{3}, Y\right)=e^{\left(\omega\left(\mathbf{p}_{1}\right)+\omega\left(\mathbf{p}_{2}\right)+\omega\left(\mathbf{p}_{3}\right)\right) Y} \\
& \quad \times \delta^{(2)}\left(\mathbf{p}_{1}-\mathbf{p}_{4}\right) \delta^{(2)}\left(\mathbf{p}_{2}-\mathbf{p}_{5}\right) \delta^{(2)}\left(\mathbf{p}_{3}-\mathbf{p}_{6}\right) \\
& \quad+\sum_{n=1}^{\infty}\left\{\prod_{i=1}^{n} \int_{0}^{y_{i-1}} d y_{i}\right. \\
& \left.\quad \times \int d^{2} \mathbf{k}_{i} e^{\left(\omega\left(\mathbf{p}_{1}\right)+\omega\left(\mathbf{p}_{2}\right)+\omega\left(\mathbf{p}_{3}\right)\right)\left(y_{i-1}-y_{i}\right)} \mathcal{O}\left(\mathbf{k}_{i}\right) \otimes\right\} \\
& \quad \times e^{\left(\omega\left(\mathbf{p}_{1}\right)+\omega\left(\mathbf{p}_{2}\right)+\omega\left(\mathbf{p}_{3}\right)\right) y_{n}} \delta^{(2)}\left(\mathbf{p}_{1}-\mathbf{p}_{4}\right) \\
& \quad \times \delta^{(2)}\left(\mathbf{p}_{2}-\mathbf{p}_{5}\right) \delta^{(2)}\left(\mathbf{p}_{3}-\mathbf{p}_{6}\right) .
\end{aligned}
$$

It is important to comment now on the infrared finiteness of this expression. The original non-forward BFKL equation is projected on a color singlet representation and reads

$$
\begin{aligned}
& \left.\left(\omega-\omega\left(\mathbf{p}_{1}\right)-\omega\left(\mathbf{p}_{2}\right)\right)\right) f_{\omega}\left(\mathbf{p}_{1}, \mathbf{p}_{2}\right)=\delta^{(2)}\left(\mathbf{p}_{1}-\mathbf{p}_{3}\right) \\
& \quad+2 \int d^{2} \mathbf{k} \xi\left(\mathbf{p}_{1}, \mathbf{p}_{2}, \mathbf{k}\right) f_{\omega}\left(\mathbf{p}_{1}+\mathbf{k}, \mathbf{p}_{2}-\mathbf{k}\right) .
\end{aligned}
$$

The solution to this equation is $\lambda$ independent in the limit $\lambda \rightarrow 0$ because each of the logarithmic divergencies generated upon integration over one of the gluons with momentum $\mathbf{k}$ is compensated by the same logarithmic $\lambda$ dependence in two of the gluon Regge trajectories. The original BKP equation (for any number of exchanged reggeons) projected on a singlet is IR finite for the same reason. For example, the divergence generated by

$$
\int d^{2} \mathbf{k} \xi\left(\mathbf{p}_{1}, \mathbf{p}_{2}, \mathbf{p}_{3}, \mathbf{k}\right) f_{\omega}\left(\mathbf{p}_{1}+\mathbf{k}, \mathbf{p}_{2}-\mathbf{k}, \mathbf{p}_{3}\right)
$$

in Eq. (3) is cancelled against the one present in $\left(\omega\left(\mathbf{p}_{1}\right)\right.$ $\left.+\omega\left(\mathbf{p}_{2}\right)\right) / 2$.

The situation is different when we project on the adjoint representation in the $t$-channel. Now the corresponding Green functions are IR divergent. Fortunately, the associated $\lambda$ dependence factorizes in a simple form. Let us go back to the BFKL equation in this new color representation, it now reads

$$
\begin{gathered}
\left.\left(\omega-\omega\left(\mathbf{p}_{1}\right)-\omega\left(\mathbf{p}_{2}\right)\right)\right) f_{\omega}\left(\mathbf{p}_{1}, \mathbf{p}_{2}\right)=\delta^{(2)}\left(\mathbf{p}_{1}-\mathbf{p}_{3}\right) \\
+\int d^{2} \mathbf{k} \xi\left(\mathbf{p}_{1}, \mathbf{p}_{2}, \mathbf{k}\right) f_{\omega}\left(\mathbf{p}_{1}+\mathbf{k}, \mathbf{p}_{2}-\mathbf{k}\right) .
\end{gathered}
$$

We can see that there is a divergent $\lambda$ dependence generated by half of the trajectories contribution $\left(\omega\left(\mathbf{p}_{1}\right)+\omega\left(\mathbf{p}_{2}\right)\right) / 2$ not compensated by the integration of $\xi$. In Refs. $[24,25]$ we have shown that in this case the gluon Green function can be written as

$$
f_{\mathrm{BFKL}}^{\text {adjoint }}\left(\mathbf{p}_{1}, \mathbf{p}_{2}, \mathrm{Y}\right)=\left(\frac{\lambda^{2}}{\sqrt{\mathbf{p}_{1}^{2} \mathbf{p}_{2}^{2}}}\right)^{\frac{\bar{\alpha}_{S} \mathrm{Y}}{2}} \widehat{f}_{\mathrm{BFKL}}^{\text {adjoint }}\left(\mathbf{p}_{1}, \mathbf{p}_{2}, \mathrm{Y}\right),
$$

where $\widehat{f}_{\mathrm{BFKL}}^{\text {adjoint }}$ is finite when $\lambda \rightarrow 0$. As we mentioned before, the usual BKP equation for three reggeized gluons is finite while the open BKP-like equation we are studying lacks the link term $\xi$ between $\mathbf{p}_{1}$ and $\mathbf{p}_{3}$. This means that the combination of trajectories $\left(\omega\left(\mathbf{p}_{1}\right)+\omega\left(\mathbf{p}_{3}\right)\right) / 2$ generates divergencies which are not cancelled. Similarly to the adjoint BFKL case these divergencies factorize at the level of the Green function in the form

$$
\begin{aligned}
& f_{\mathrm{BKP}}^{\text {adjoint }}\left(\mathbf{p}_{1}, \mathbf{p}_{2}, \mathbf{p}_{3}, \mathrm{Y}\right) \\
& =\left(\frac{\lambda^{2}}{\sqrt{\mathbf{p}_{1}^{2} \mathbf{p}_{3}^{2}}}\right)^{\frac{\bar{\alpha}_{S} \mathrm{Y}}{2}} \widehat{f}_{\mathrm{BKP}}^{\text {adjoint }}\left(\mathbf{p}_{1}, \mathbf{p}_{2}, \mathbf{p}_{3}, \mathrm{Y}\right) .
\end{aligned}
$$

It is the infrared finite function $\widehat{f_{\mathrm{BKP}}}$ the one we will study in depth in this work. Let us now indicate that the $\lambda$ independence can be achieved order-by-order in a coupling expansion but this is not very useful for our purposes. We work instead with an effective field theory where each Feynman diagram is not infrared finite. Finiteness is achieved only after summing up all contributing effective Feynman diagrams to all those orders being numerically significant. We explained this method in detail in Refs. [24,25]. We describe the main features emerging from our solution in the following section.

\section{Solution to the open spin chain}

In this section we evaluate the solution to the OSC and compare it to that in the closed case. It is instructive to discuss 

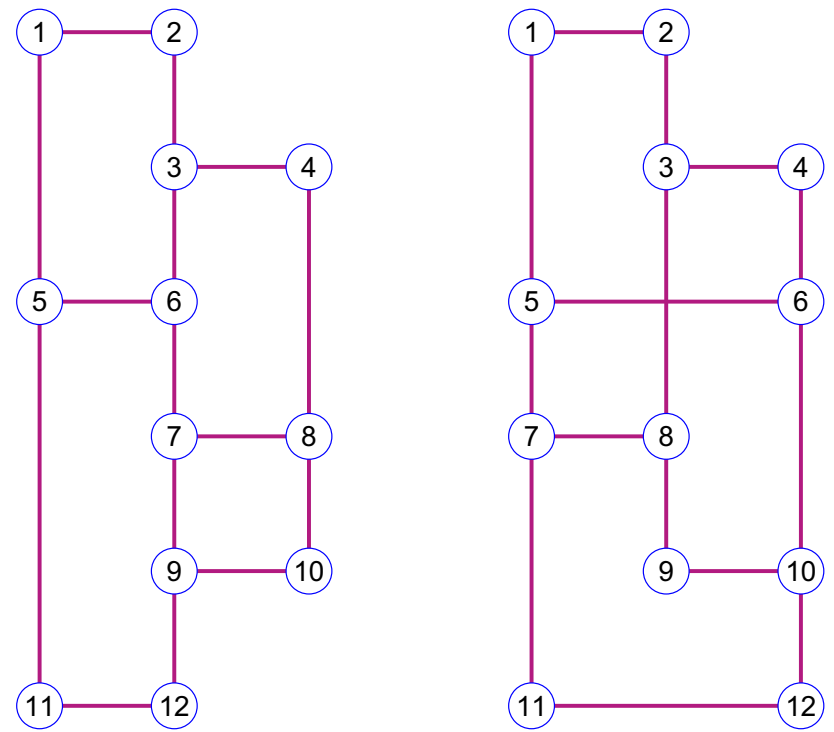

Fig. 2 Two typical diagrams with six rungs. Open spin chain topology to the left (labeled $\{\mathrm{L}, \mathrm{R}, \mathrm{L}, \mathrm{R}, \mathrm{R}, \mathrm{L}\}$ ) and closed spin chain topology to the right (labeled $\{\mathrm{L}, \mathrm{R}, \mathrm{M}, \mathrm{L}, \mathrm{R}, \mathrm{M}\}$ )

first a few key points regarding the type of diagrams one encounters in the two cases.

We will always have three Reggeons labeled (1), (2) and (3) vertically aligned and ordered such that (1) is to the left and (3) to the right (see the plot at the right hand side of Fig. 1). In the CSC case each Reggeon is allowed to interact with any of the other two via gluon exchange. We will call these gluon exchanges rungs following the nomenclature associated to generalised ladder diagrams. We will label a rung between Reggeons (1) and (2) by L (left), a rung between Reggeons (2) and (3) by R (right) and a rung between Reggeons (1) and (3) by M. As an example, in Fig. 2 we can see two six-rung ladder diagrams, one for the OSC case (to the left) and one for the closed case (to the right). In the following, without any loss of generality, we will assume that in the OSC case the Reggeons that cannot interact directly via a gluon exchange are the (1) and (3) (Fig. 3).

From Fig. 2, it is evident that an ordered list of the labels that correspond to the rungs of a ladder diagram defines it unambiguously. In our diagrams we only have two types of nodes: those with two attached legs and those with three. There are only six of the first type which correspond to threepoint vertices where we have removed one propagator carrying one of the external momenta $\mathbf{p}_{i}$. The adjacency matrix of a given diagram is the square matrix with off-diagonal elements being the number of lines connecting the vertex $i$ with the vertex $j$. Its diagonal elements are zero. Using a labelling system as described above allows the fast computation of the adjacency matrix and adjacency list for any ladder diagram opening the road for more detailed studies of the diagrammatic topologies that appear after each iteration
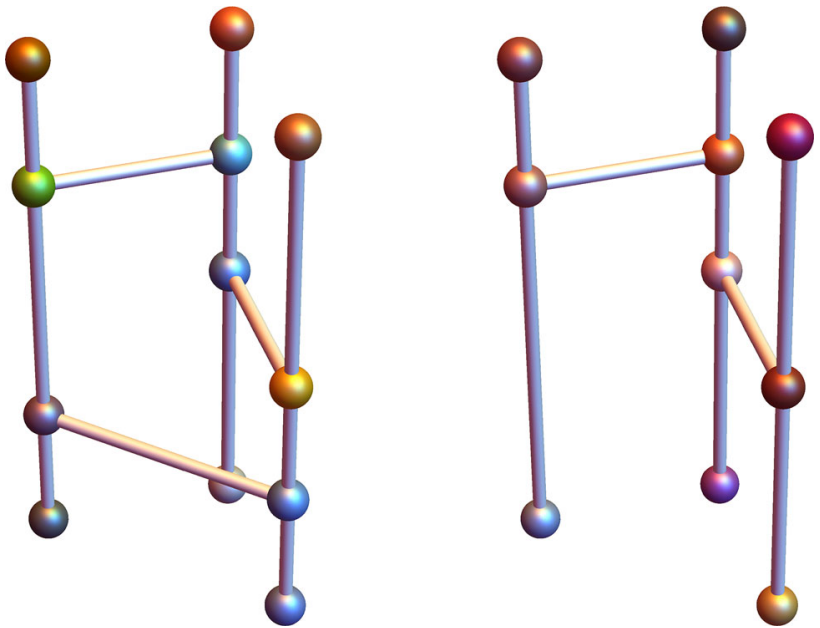

Fig. 3 A closed spin chain diagram (left) has a cylindrical symmetry and cannot be embedded on a plane. Conversely, an open spin diagram (right), has no cylindrical symmetry and is planar

of the kernel. As an example we now show the adjacency matrices associated to the diagrams in Fig. 2. For the CSC example,

$\left(\begin{array}{llllllllllll}0 & 1 & 0 & 0 & 1 & 0 & 0 & 0 & 0 & 0 & 0 & 0 \\ 1 & 0 & 1 & 0 & 0 & 0 & 0 & 0 & 0 & 0 & 0 & 0 \\ 0 & 1 & 0 & 1 & 0 & 0 & 0 & 1 & 0 & 0 & 0 & 0 \\ 0 & 0 & 1 & 0 & 0 & 1 & 0 & 0 & 0 & 0 & 0 & 0 \\ 1 & 0 & 0 & 0 & 0 & 1 & 1 & 0 & 0 & 0 & 0 & 0 \\ 0 & 0 & 0 & 1 & 1 & 0 & 0 & 0 & 0 & 1 & 0 & 0 \\ 0 & 0 & 0 & 0 & 1 & 0 & 0 & 1 & 0 & 0 & 1 & 0 \\ 0 & 0 & 1 & 0 & 0 & 0 & 1 & 0 & 1 & 0 & 0 & 0 \\ 0 & 0 & 0 & 0 & 0 & 0 & 0 & 1 & 0 & 1 & 0 & 0 \\ 0 & 0 & 0 & 0 & 0 & 1 & 0 & 0 & 1 & 0 & 0 & 1 \\ 0 & 0 & 0 & 0 & 0 & 0 & 1 & 0 & 0 & 0 & 0 & 1 \\ 0 & 0 & 0 & 0 & 0 & 0 & 0 & 0 & 0 & 1 & 1 & 0\end{array}\right)$,

and for the open,

$$
\left(\begin{array}{llllllllllll}
0 & 1 & 0 & 0 & 1 & 0 & 0 & 0 & 0 & 0 & 0 & 0 \\
1 & 0 & 1 & 0 & 0 & 0 & 0 & 0 & 0 & 0 & 0 & 0 \\
0 & 1 & 0 & 1 & 0 & 1 & 0 & 0 & 0 & 0 & 0 & 0 \\
0 & 0 & 1 & 0 & 0 & 0 & 0 & 1 & 0 & 0 & 0 & 0 \\
1 & 0 & 0 & 0 & 0 & 1 & 0 & 0 & 0 & 0 & 1 & 0 \\
0 & 0 & 1 & 0 & 1 & 0 & 1 & 0 & 0 & 0 & 0 & 0 \\
0 & 0 & 0 & 0 & 0 & 1 & 0 & 1 & 1 & 0 & 0 & 0 \\
0 & 0 & 0 & 1 & 0 & 0 & 1 & 0 & 0 & 1 & 0 & 0 \\
0 & 0 & 0 & 0 & 0 & 0 & 1 & 0 & 0 & 1 & 0 & 1 \\
0 & 0 & 0 & 0 & 0 & 0 & 0 & 1 & 1 & 0 & 0 & 0 \\
0 & 0 & 0 & 0 & 1 & 0 & 0 & 0 & 0 & 0 & 0 & 1 \\
0 & 0 & 0 & 0 & 0 & 0 & 0 & 0 & 1 & 0 & 1 & 0
\end{array}\right) .
$$

We will make use of this representation of the Feynman diagrams in the following section.

In Ref. [16], we developed a Monte Carlo code to compute the Odderon Green function based on our experience with 
BFKLex [24-30]. In principle, a straightforward approach would be to modify as little as possible our existing code for the CSC case and run it for the OSC one. However, we realized at an early stage of this work that we would need to develop a code tailored especially for the OSC case. This was mainly due to the fact that convergence now was not as fast as in the CSC case and therefore we had to optimise our code as much as possible. In the following we will describe some key issues regarding our numerical approach, for more details we refer the reader to Ref. [16].

We want to compute Eq. (9) in order to obtain $f\left(\mathbf{p}_{1}, \mathbf{p}_{2}\right.$, $\mathbf{p}_{3}, Y$ ) for some given momenta configuration $\mathbf{p}_{1}, \mathbf{p}_{2}, \mathbf{p}_{3}, \mathbf{p}_{4}$, $\mathbf{p}_{5}, \mathbf{p}_{6}$ and for varying rapidity $Y$. To be specific, we will consider the following values for the transverse momenta (they are shown in polar coordinates, the first entry stands for the modulus of the momentum and the second one for the azimuthal angle):

$$
\begin{aligned}
& \mathbf{p}_{1}=(10,0) \\
& \mathbf{p}_{2}=(20, \pi) \\
& \mathbf{p}_{3}=\mathbf{q}-\mathbf{p}_{1}-\mathbf{p}_{2} ; \\
& \mathbf{p}_{4}=(20,0) ; \\
& \mathbf{p}_{5}=(25, \pi) \\
& \mathbf{p}_{6}=\mathbf{q}-\mathbf{p}_{4}-\mathbf{p}_{5}
\end{aligned}
$$

The units of the moduli in the two-vectors above are expressed in $\mathrm{GeV}$. The momentum transfer $\mathbf{q}$ satisfies

$\mathbf{p}_{1}+\mathbf{p}_{2}+\mathbf{p}_{3}=\mathbf{q}=\mathbf{p}_{3}+\mathbf{p}_{4}+\mathbf{p}_{6}$

and takes the following values: $\mathbf{q}=\{(4,0),(17,0),(31,0)$, $(107,0)\}$. Finally, we vary the rapidity $Y$ from 1 to 5.5 units and in one case up to 6.5 (Fig. 9).

As in the CSC case, non-zero contributions to $f\left(\mathbf{p}_{1}, \mathbf{p}_{2}\right.$, $\mathbf{p}_{3}, Y$ ) will appear only after we consider two rungs. Any given diagram with $i$ rungs, once iterated, will generate two new diagrams each with $i+1$ rungs. This leads to a complete binary tree structure:

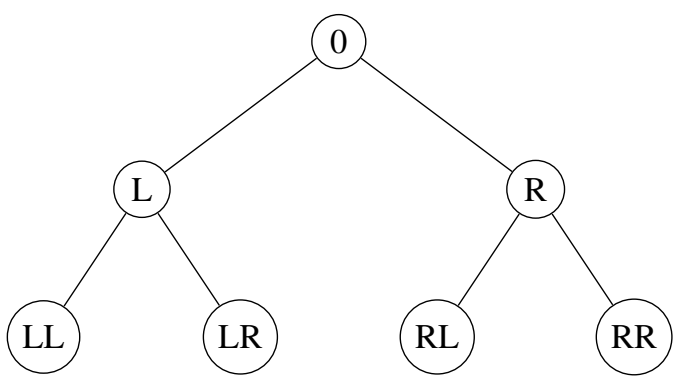

Two of the momenta integrations are trivial since there are three Dirac delta functions to be fulfilled. However, the junctions (see Ref. [16]) in the OSC are only two: $\mathcal{J}_{\mathrm{LR}}, \mathcal{J}_{\mathrm{RL}}$. Lastly, any diagram with no junction, in other words, any diagram $\mathcal{J}_{\mathrm{Q}}$ with $\mathrm{Q}$ being a sequence of only $\mathrm{L}$ or only $\mathrm{R}$ is
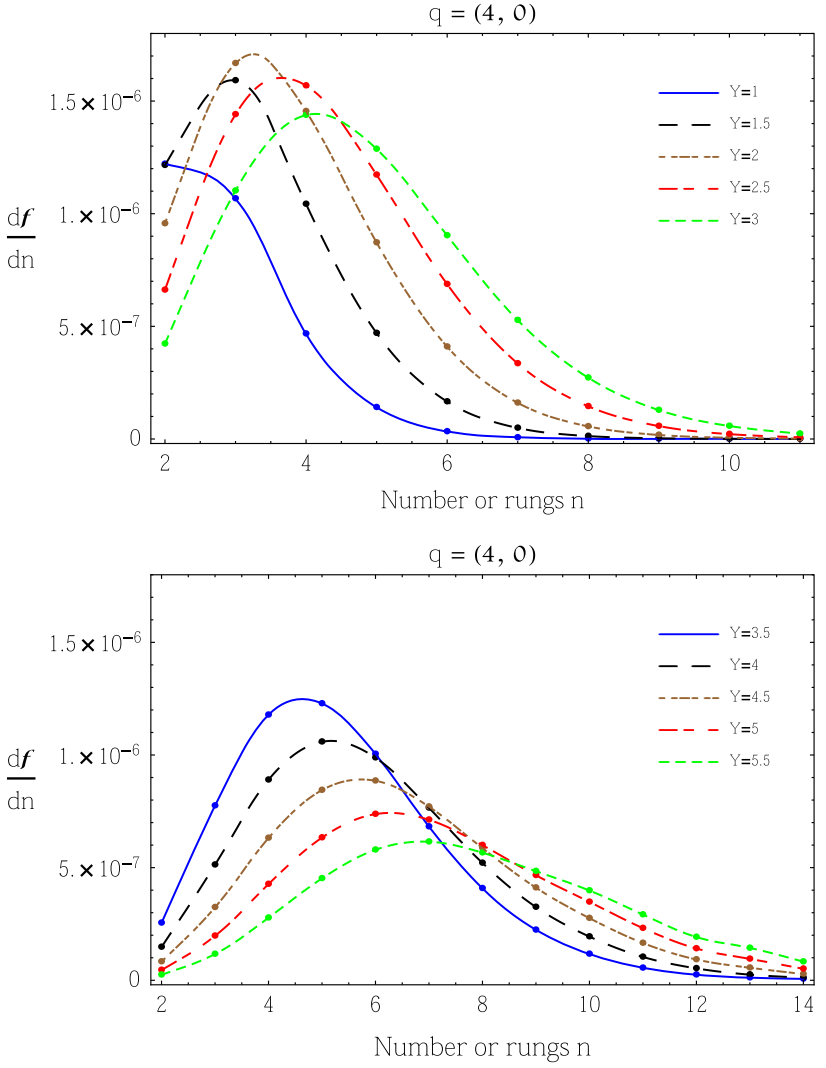

Fig. 4 Multiplicity plot for $q=4 \mathrm{GeV}$ in the closed spin chain

zero. This becomes clear since all $\mathbf{p}_{i}$ are chosen to be different from each other and hence none of the Dirac delta functions in the initial condition is fulfilled.

In the following we are presenting four multiplicity (in the sense of counting the number of rungs in the diagram) plots, two of them (smaller and larger rapidities) with momentum transfer, $\mathbf{q}=(4,0)$ (Fig. 5) and two more for $\mathbf{q}=(31,0)$ (Fig. 7). We compare these to the corresponding plots from the CSC, Figs. 4 and 6 respectively.

We verify anew that the contributions from each iteration for a given $Y$ once plotted versus $n$, where $n$ is the number of rungs or iterations, follow a Poisson-like distribution, similarly to the CSC. The similarities between the open and CSC do not end there. In both cases we observe a global maximum at some $n$ and then a decrease as $n$ increases. The Green function is noticeably smaller when the total momentum transfer is larger. Furthermore, the peak of the distribution moves similarly to larger values of $n$ for both values of $\mathbf{q}$ as $Y$ increases, whereas its height gets lower and the distributions are much broader. As $|\mathbf{q}|$ increases the position of the peak shifts to a larger $n$.

There is an interesting qualitative difference between the closed and OSC cases. Let us focus on the bottom plots in Figs. 4 and 5. We observe that in the OSC the decrease in the value of the maximal point of the distribution takes place 

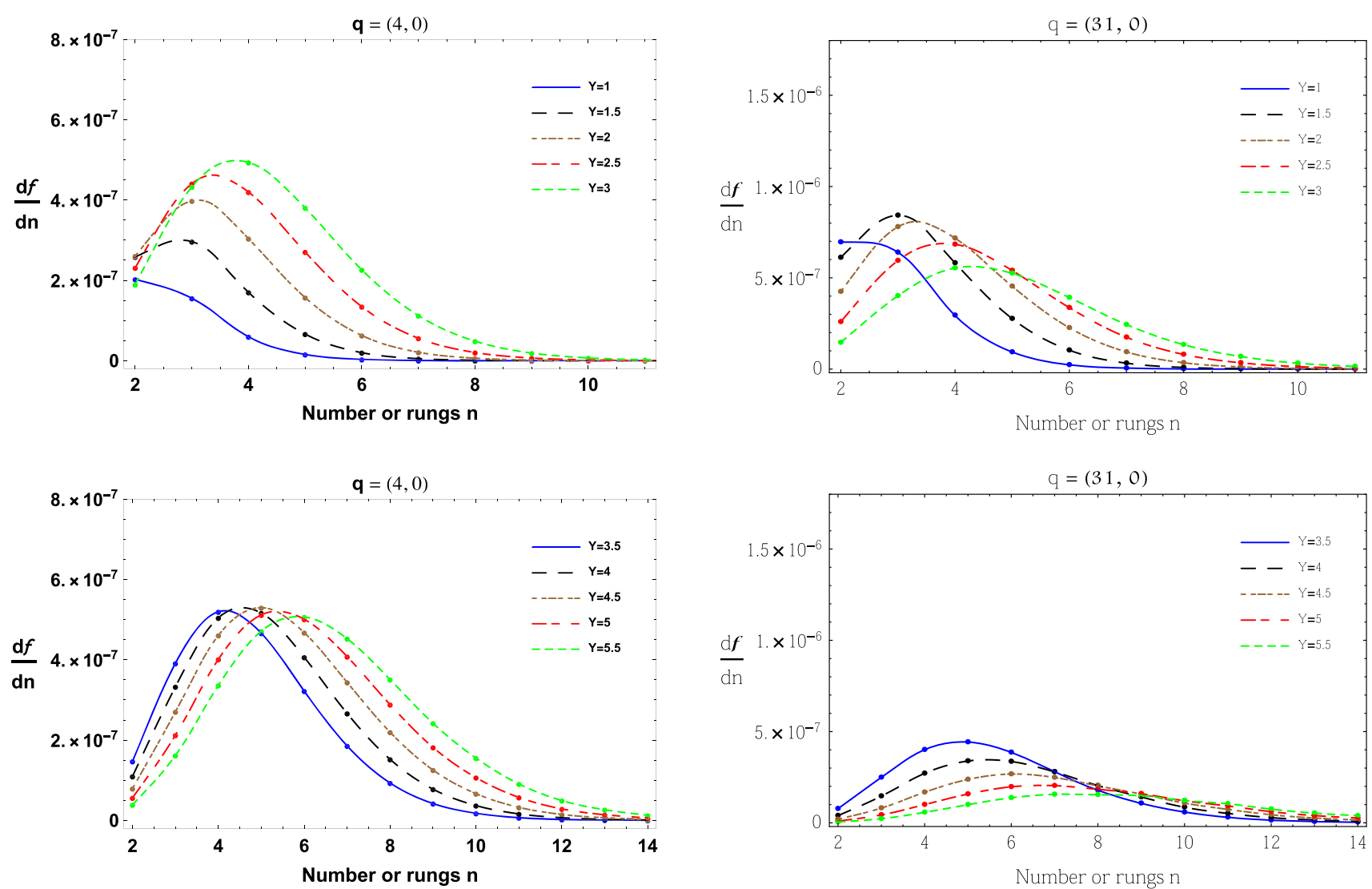

Fig. 5 Multiplicity plot for $q=4 \mathrm{GeV}$ in the open spin chain

very slowly as we vary $Y$. This is different to the CSC configuration where the distribution broadens very quickly with $Y$. This sudden broadening of the multiplicity distribution is a distinct signal of the cylinder topology of the contributing effective Feynman diagrams (Fig. 3).

The six-point gluon Green functions correspond to the areas under the distributions just described. Their dependence with $Y$ is drawn in Figs. 8 and 9. While for the CSC we see that the energy $(\ln Y)$ dependence plot of the Green function has its peak at relatively small rapidities $(Y<3)$ for both $\mathbf{q}=(4,0)$ and $\mathbf{q}=(31,0)$ and then it starts to decrease noticeably fast, for the OSC on the other hand, we see that for $\mathbf{q}=(4,0)$ the curve increases monotonically. If we now increase the momentum transfer, we see that for $\mathbf{q}=(17,0)$ (brown dashed line) the curve rises although much slower than for $\mathbf{q}=(4,0)$. If we increase further to $\mathbf{q}=(31,0)$ (red dashed line) we see that for $Y>6$ (notice that we have pushed the upper limit of $Y$ here for this plot to 6.5 units) the curve seems to decrease. An even further increase to $\mathbf{q}=(107,0)$ (orange dashed line) gives us a plot with a clear maximum at around $Y \sim 3.4$.

To summarize, we find a similar qualitative behavior between the closed and open Green function for the main features we could assess in our numerical analysis. However,

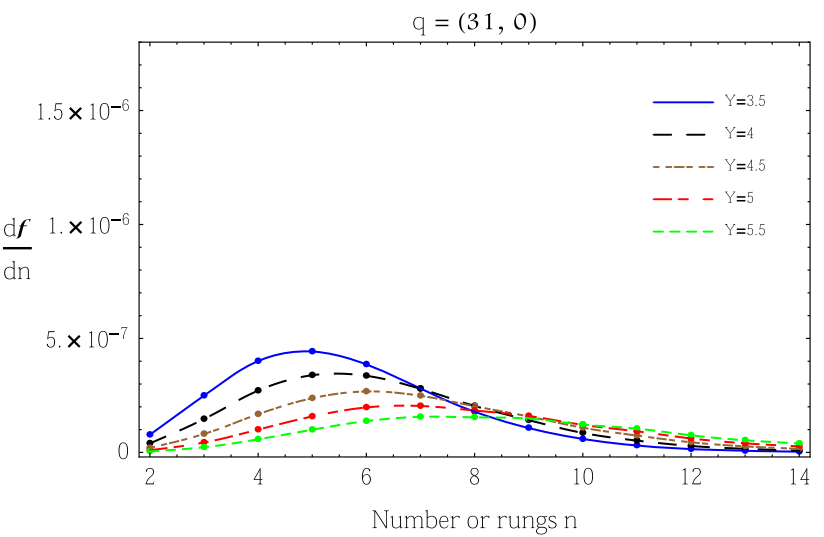

Fig. 6 Multiplicity plot for $q=31 \mathrm{GeV}$ in the closed spin chain

the CSC Green function seems to approach the asymptotia much faster than the OSC one. Moreover, the smaller the momentum transfer, the more amplified this trend is. It will be very interesting to see what happens when one integrates the Green function with impact factors which is a crucial step to construct the full $n$-point amplitudes in the supersymmetric theory (Ref. [31] offers an interesting review on the systematics behind these calculations), but this is a question beyond the scope of this work. Let us now conclude with a section devoted to a study of the graph complexity associated to the Feynman diagrams contributing to the gluon Green function.

\section{Graph complexity}

Let us highlight some aspects of graph theory which we have used in our study of the Reggeon spin chains (some reviews on the subject can be found in [32,33]). Our graphs $\mathcal{G}$ consist of a set of vertices $V$ and propagators (edges) $P, \mathcal{G}=(V, P)$. All the diagrams are connected since we do not allow for $\mathbf{p}_{1}$ to be equal to $\mathbf{p}_{4}, \mathbf{p}_{2}$ to $\mathbf{p}_{5}$, or $\mathbf{p}_{3}$ to $\mathbf{p}_{6}$. Only for connected graphs we can define spanning trees, which are those paths within the graph which connect all its vertices without any cycles. 

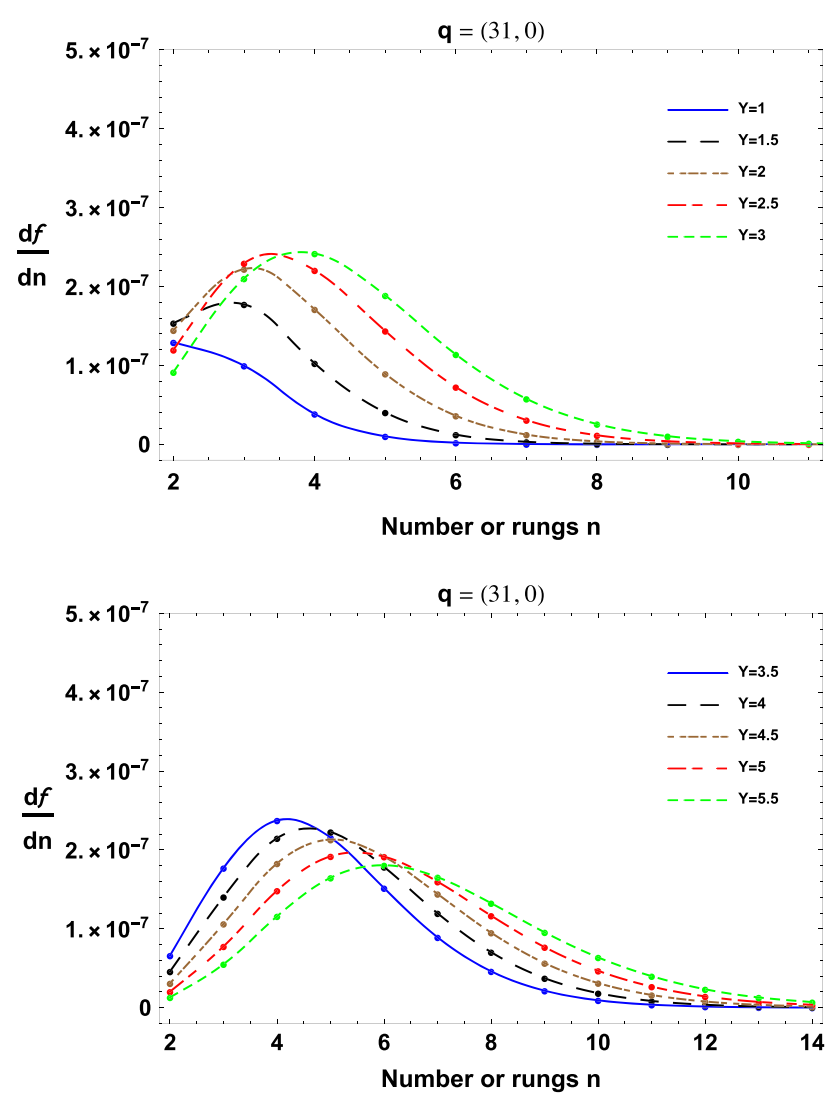

Fig. 7 Multiplicity plot for $q=31 \mathrm{GeV}$ in the open spin chain

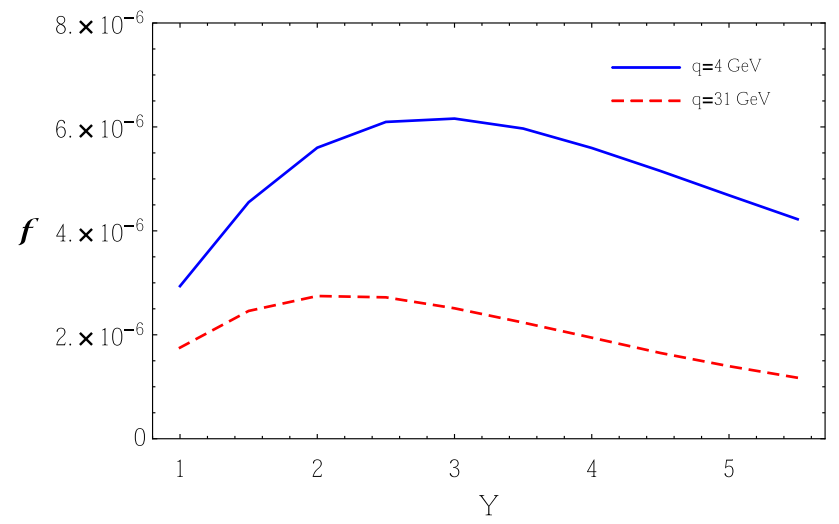

Fig. 8 Energy dependence of $f$ for $q=4 \mathrm{GeV}$ (blue continuous line) and $q=31 \mathrm{GeV}$ (red dashed line) for the closed spin chain

The complexity of an undirected connected graph corresponds to the number of all possible spanning trees of the graph $\mathcal{G}$. The degree of a particular vertex $i, d_{\mathcal{G}}(i)$, is the number of half-edges which are in contact with it. Since we can connect two or three propagators to each node then the vertices can have degree 2 or 3 .

If $|V|$ is the number of vertices in $\mathcal{G}$ then we can define the degree matrix of $\mathcal{G}, D_{\mathcal{G}}$, as the diagonal $|V| \times|V|$ square matrix with diagonal elements $d_{\mathcal{G}}(i)$. Its matrix elements are of the form $D_{\mathcal{G}}(i, j)=d_{\mathcal{G}}(i) \delta_{i j}$. As mentioned in a previous

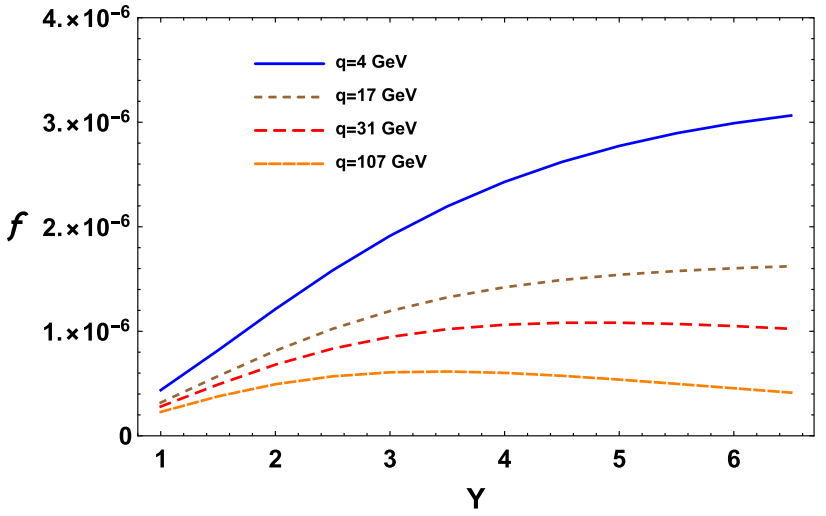

Fig. 9 Energy dependence of $f$ for $q=4 \mathrm{GeV}$ (blue continuous line), $q=31 \mathrm{GeV}$ (red dashed line), $q=17 \mathrm{GeV}$ (brown dashed line) and $q=107 \mathrm{GeV}$ (green dashed line) for the open spin chain

section, the adjacency matrix of $\mathcal{G}, A_{\mathcal{G}}$, is the $|V| \times|V|$ square matrix with off-diagonal elements being the number of propagators connecting the vertex $i$ with the vertex $j$. The Laplacian matrix corresponds to the difference of these two matrices: $L_{\mathcal{G}}=D_{\mathcal{G}}-A_{\mathcal{G}}$. As we will see, these matrices carry the topological information of the graph. For the sake of clarity, we explicitly write the Laplacian matrices associated to the diagrams in Fig. 2. In the CSC we have

$$
\left(\begin{array}{cccccccccccc}
2 & -1 & 0 & 0 & -1 & 0 & 0 & 0 & 0 & 0 & 0 & 0 \\
-1 & 2 & -1 & 0 & 0 & 0 & 0 & 0 & 0 & 0 & 0 & 0 \\
0 & -1 & 3 & -1 & 0 & 0 & 0 & -1 & 0 & 0 & 0 & 0 \\
0 & 0 & -1 & 2 & 0 & -1 & 0 & 0 & 0 & 0 & 0 & 0 \\
-1 & 0 & 0 & 0 & 3 & -1 & -1 & 0 & 0 & 0 & 0 & 0 \\
0 & 0 & 0 & -1 & -1 & 3 & 0 & 0 & 0 & -1 & 0 & 0 \\
0 & 0 & 0 & 0 & -1 & 0 & 3 & -1 & 0 & 0 & -1 & 0 \\
0 & 0 & -1 & 0 & 0 & 0 & -1 & 3 & -1 & 0 & 0 & 0 \\
0 & 0 & 0 & 0 & 0 & 0 & 0 & -1 & 2 & -1 & 0 & 0 \\
0 & 0 & 0 & 0 & 0 & -1 & 0 & 0 & -1 & 3 & 0 & -1 \\
0 & 0 & 0 & 0 & 0 & 0 & -1 & 0 & 0 & 0 & 2 & -1 \\
0 & 0 & 0 & 0 & 0 & 0 & 0 & 0 & 0 & -1 & -1 & 2
\end{array}\right),
$$

and in the OSC case,

$$
\left(\begin{array}{cccccccccccc}
2 & -1 & 0 & 0 & -1 & 0 & 0 & 0 & 0 & 0 & 0 & 0 \\
-1 & 2 & -1 & 0 & 0 & 0 & 0 & 0 & 0 & 0 & 0 & 0 \\
0 & -1 & 3 & -1 & 0 & -1 & 0 & 0 & 0 & 0 & 0 & 0 \\
0 & 0 & -1 & 2 & 0 & 0 & 0 & -1 & 0 & 0 & 0 & 0 \\
-1 & 0 & 0 & 0 & 3 & -1 & 0 & 0 & 0 & 0 & -1 & 0 \\
0 & 0 & -1 & 0 & -1 & 3 & -1 & 0 & 0 & 0 & 0 & 0 \\
0 & 0 & 0 & 0 & 0 & -1 & 3 & -1 & -1 & 0 & 0 & 0 \\
0 & 0 & 0 & -1 & 0 & 0 & -1 & 3 & 0 & -1 & 0 & 0 \\
0 & 0 & 0 & 0 & 0 & 0 & -1 & 0 & 3 & -1 & 0 & -1 \\
0 & 0 & 0 & 0 & 0 & 0 & 0 & -1 & -1 & 2 & 0 & 0 \\
0 & 0 & 0 & 0 & -1 & 0 & 0 & 0 & 0 & 0 & 2 & -1 \\
0 & 0 & 0 & 0 & 0 & 0 & 0 & 0 & -1 & 0 & -1 & 2
\end{array}\right) .
$$

The Laplacian matrix is very useful to compute the complexity of each Reggeon graph in a simple way. The key result 
is the well-known Matrix-Tree theorem [34] which is one of most fundamental results in combinatorial theory and states that the complexity of a graph corresponds to the value of the determinant of the Laplacian matrix once we remove one of its rows and one of its columns (the determinant of any of its principal minors). The complexity does not depend on a possible ordering of the vertices. Applying the Matrix-Tree theorem to the two graphs in Fig. 2 we find that the number of possible spanning trees in the CSC example is 532 and in the open case 463 . For a fixed number of rungs the number of graphs in the CSC case is much larger than in the OSC configuration. As an example we show the relevant topologies along with their corresponding complexity for four rungs in Fig. 10 for the OSC and in Fig. 11 for the OSC. It is natural to find that the OSC topologies are contained in the CSC possible diagrams. Moreover, the Pomeron ladder topology where all rungs are connecting the same pair of Reggeons appears twice in $\operatorname{OSC}(\{\mathrm{L}, \mathrm{L}, \mathrm{L}, \mathrm{L}\}$ and $\{\mathrm{R}, \mathrm{R}, \mathrm{R}, \mathrm{R}\})$ and thrice in $\operatorname{CSC}(\{\mathrm{L}, \mathrm{L}, \mathrm{L}, \mathrm{L}\},\{\mathrm{M}, \mathrm{M}, \mathrm{M}, \mathrm{M}\}$ and $\{\mathrm{R}, \mathrm{R}, \mathrm{R}, \mathrm{R}\})$. It is interesting to note that the Pomeron ladder has always the maximal complexity $t(n)$ of all the diagram topologies for any given number $n$ of rungs. For example, in Figs. 10 and $11 t(n)=56$ for the Pomeron ladder topologies (see top left diagrams in both figures). The complexity of the Pomeron ladder is equal to the number of spanning trees in a $2 \times n$ grid which is given by

$t(n)=4 t(n-1)-t(n-2)$,

with $\mathrm{t}(0)=0, \mathrm{t}(1)=1$ or, equivalently,

$t(n)=\frac{(2+\sqrt{3})^{n}-(2-\sqrt{3})^{n}}{2 \sqrt{3}}$.

It will be relevant to investigate whether similar relations hold for the sub-leading in complexity topologies in both the OSC and CSC cases.

We have studied what is the contribution to the gluon Green function from different graph complexities. Of course each effective Feynman diagram carries a certain statistical weight in our Monte Carlo method to generate the solution to the BKP equation which is related to the particular number of reggeized gluon propagators and squared Lipatov's vertices present in a graph with $n$ rungs. It is clear that the average complexity of the graphs grows with $n$. We have found an interesting scaling behavior obtained by the following method. Let us consider all those diagrams with the same complexity for a fixed number of rungs. Then we evaluate the average weight of their contribution to the gluon Green function as a complexity class. All of this is calculated for a fixed value of the strong coupling and rapidity. We show a characteristic sample of our results in Fig. 12 for the CSC and in Fig. 13 for the OSC.
Per complexity value, those corresponding to a lower number of rungs have a bigger mean weight but their number is smaller. The amount of possible complexity values grows very fast with $n$. This is not surprising since the number of nodes in the graphs is proportional to the number of rungs. What we find very remarkable is that for the larger values of complexity in each set with the same $n$ all different complexities contribute with a very similar weight to the solution of the BKP equation. This trend is independent of having a closed or open Reggeon graph and for different values of the available parameters. We suspect that this "complexity democracy" is likely to be related to the underlying integrability found by Lipatov. To establish the definite link is the subject of some of our current investigations.

\section{Conclusions and outlook}

The high energy limit of scattering amplitudes in field theory and gravity [35-38] has a very rich structure. In QCD an integrable structure arises when the center-of-mass energy is much larger than any Mandelstam invariant in the scattering process. This integrability, realized in coordinate representation, is related to the mapping to a Heisenberg ferromagnet with non-compact spins and periodic boundary conditions (closed spin chain). Recently, we have shown how to solve this system in momentum and rapidity space making use of Monte Carlo integration techniques. Our solution calculates the gluon Green function exactly and is general, it does not rely on any choice of normalization of the composite state wave function. For the three reggeon case it corresponds to the solution to the perturbative Odderon.

More recently, Lipatov found a new integrable spin chain, in this case open, in the context of the calculation of scattering amplitudes in the $N=4$ supersymmetric Yang-Mills theory. This new open spin chain structure is important since it is the most complicated piece to evaluate when dealing with the multi-Regge limit of scattering amplitudes in Mandelstam regions for amplitudes with a large number of legs. It is very important to understand it in detail in order to advance in our knowledge of the all-orders structure of amplitudes in a theory which allows for a smooth matching between the weak and the strong coupling limits [39-47].

In the present work we have solved the open spin chain problem exactly again using Monte Carlo integration. The infrared divergencies present in the calculation have been shown to factorize and we have investigated the infrared finite part of the gluon Green function. We have shown that it decreases with energy, as in the closed spin chain case although this behavior is delayed as the momentum transfer is reduced. Our results will allow to fix some of the uncertainties present when evaluating the eight-point amplitude in exact kinematics. For this it is still needed to integrate our 

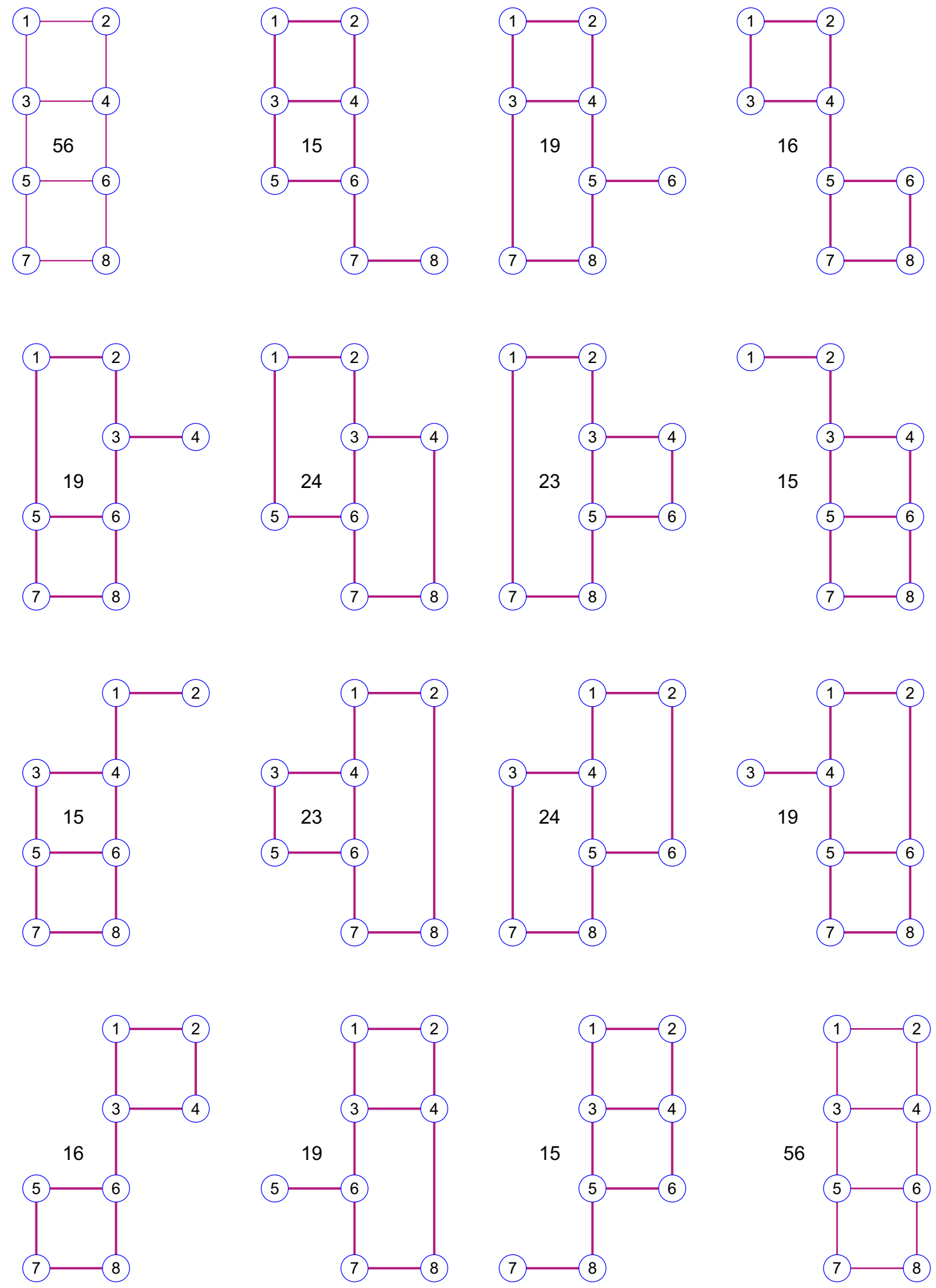

Fig. 10 Open spin chain topologies with 4 rungs. The labels of the topologies from left to right and top to bottom are $\{\mathrm{L}, \mathrm{L}, \mathrm{L}, \mathrm{L}\},\{\mathrm{L}$, $\mathrm{L}, \mathrm{L}, \mathrm{R}\},\{\mathrm{L}, \mathrm{L}, \mathrm{R}, \mathrm{L}\},\{\mathrm{L}, \mathrm{L}, \mathrm{R}, \mathrm{R}\},\{\mathrm{L}, \mathrm{R}, \mathrm{L}, \mathrm{L}\},\{\mathrm{L}, \mathrm{R}, \mathrm{L}, \mathrm{R}\},\{\mathrm{L}$,

$\mathrm{R}, \mathrm{R}, \mathrm{L}\},\{\mathrm{L}, \mathrm{R}, \mathrm{R}, \mathrm{R}\},\{\mathrm{R}, \mathrm{L}, \mathrm{L}, \mathrm{L}\},\{\mathrm{R}, \mathrm{L}, \mathrm{L}, \mathrm{R}\},\{\mathrm{R}, \mathrm{L}, \mathrm{R}, \mathrm{L}\},\{\mathrm{R}$, $\mathrm{L}, \mathrm{R}, \mathrm{R}\},\{\mathrm{R}, \mathrm{R}, \mathrm{L}, \mathrm{L}\},\{\mathrm{R}, \mathrm{R}, \mathrm{L}, \mathrm{R}\},\{\mathrm{R}, \mathrm{R}, \mathrm{R}, \mathrm{L}\},\{\mathrm{R}, \mathrm{R}, \mathrm{R}, \mathrm{R}\}$. The corresponding complexity for each graph is shown 

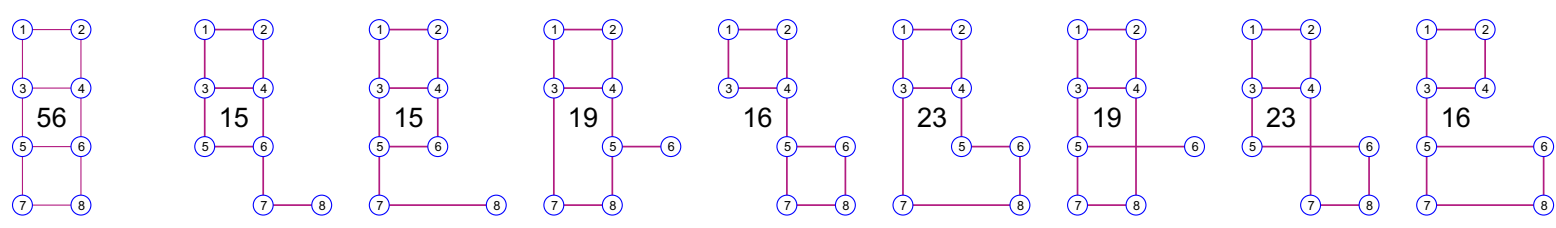

(1)-(4) (3)
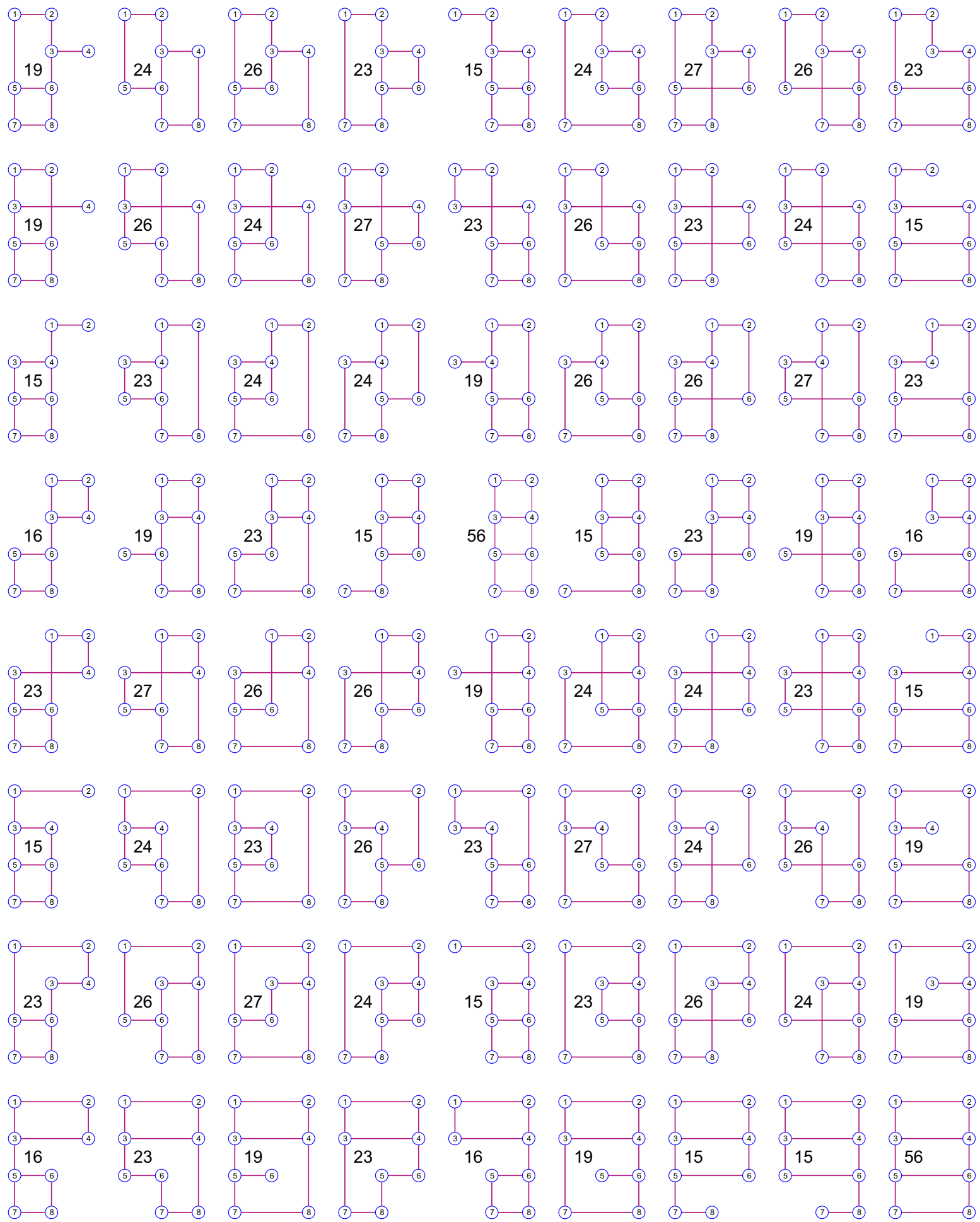

Fig. 11 Closed spin chain topologies with $n=4$ rungs. There are in total $3^{n}=81$ topologies. Trivially, the set of topologies found in the OSC (Fig. 10 ) is contained as a subset here. The corresponding complexity for each graph is shown 


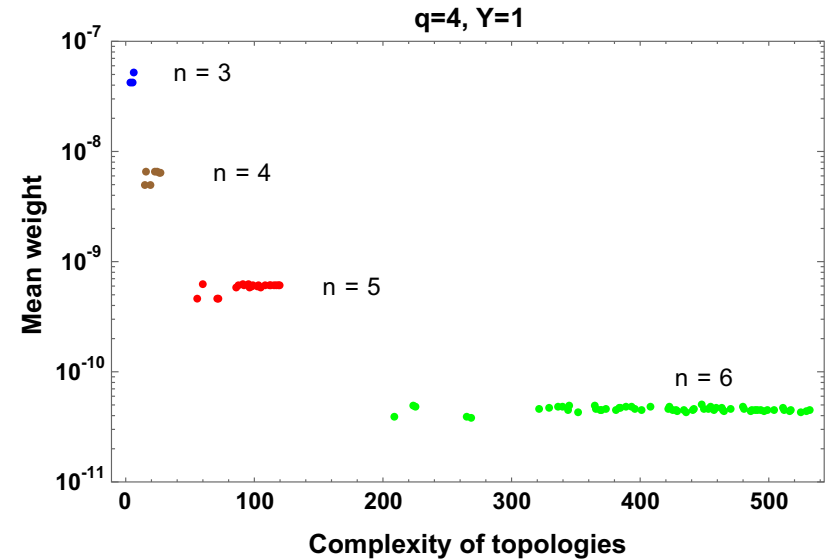

(a) Closed spin chain, $Y=1$.

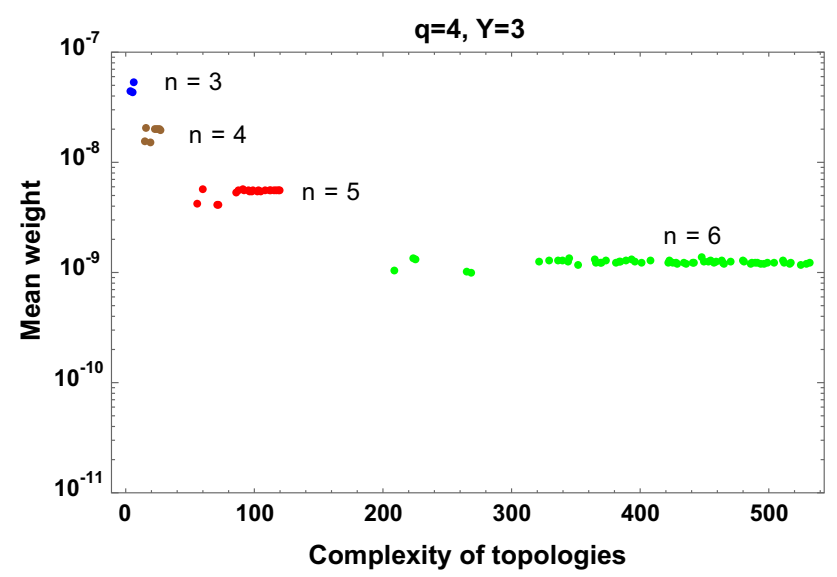

(b) Closed spin chain, $Y=3$.

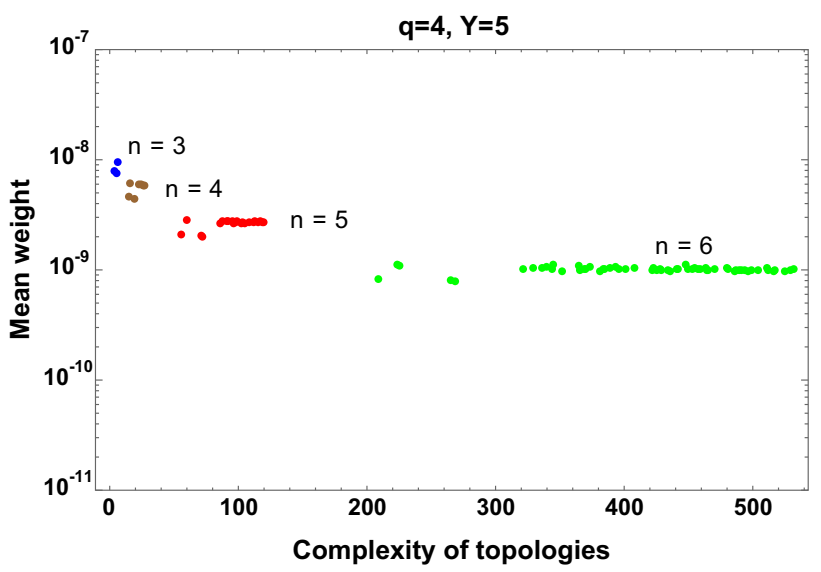

(c) Closed spin chain, $Y=5$.

Fig. 12 Average weight per complexity class for closed Reggeon webs

results over impact factors and this will be the subject of our future work. Our techniques are valid for any number of reggeized gluons and apply also at next-to-leading and higher orders [48]. This implies that they will be important for the evaluation of the general $n$-point amplitudes in exact kinematics [49-51].

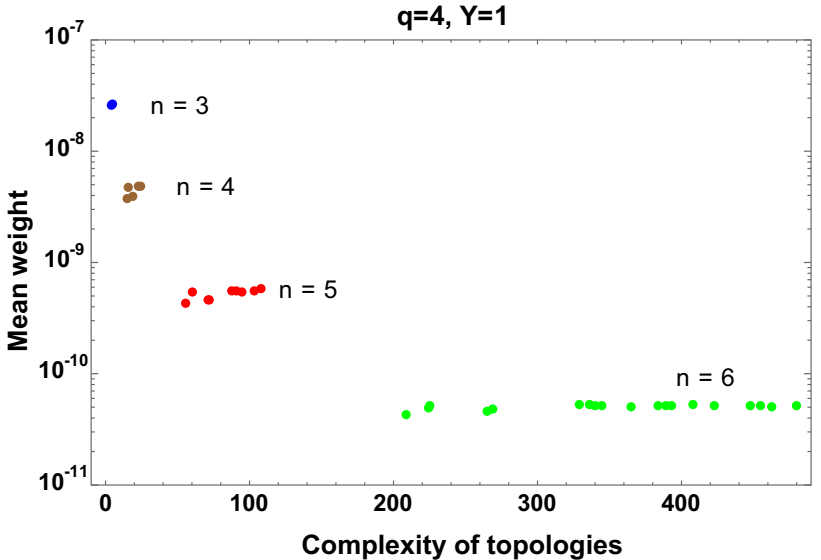

(a) Open spin chain, $Y=1$.

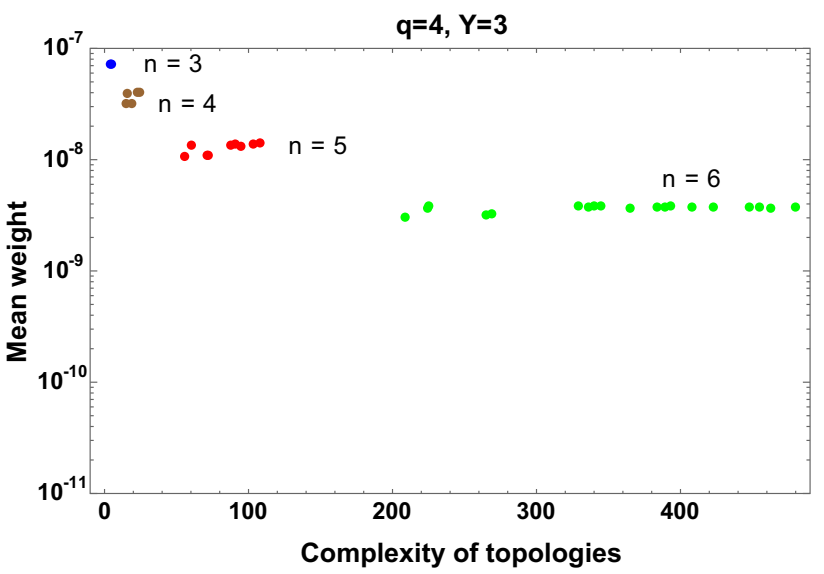

(b) Open spin chain, $Y=3$.

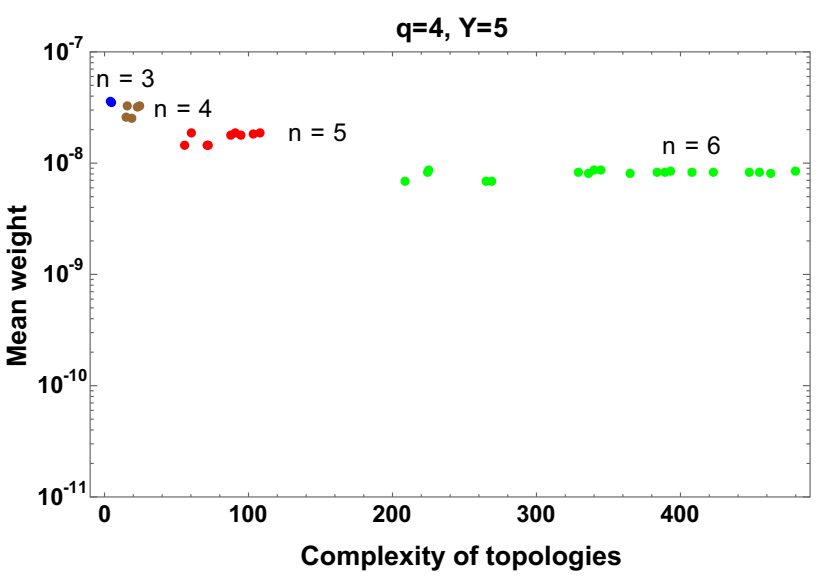

(c) Open spin chain, $Y=5$.

Fig. 13 Average weight per complexity class for open Reggeon webs

As a by-product of our work, we have found an intriguing scaling behavior of what we can call weighted complexity of the Feynman graphs contributing to the gluon Green function. The complexity of a diagram is a well-defined quantity in graph theory. We have evaluated the average weight per topology in the sense of its total contribution to the gluon 
Green function and found that it is approximately constant for a fixed number of rungs of the class of effective Feynman diagrams. This "complexity democracy" is very likely related to the integrability found by Lipatov. It will be interesting to find the precise link between both concepts.

Acknowledgements We acknowledge support from the Spanish Government Grants FPA2015-65480-P, FPA2016-78022-P and Spanish MINECO Centro de Excelencia Severo Ochoa Programme (SEV-20160597).

Open Access This article is distributed under the terms of the Creative Commons Attribution 4.0 International License (http://creativecomm ons.org/licenses/by/4.0/), which permits unrestricted use, distribution, and reproduction in any medium, provided you give appropriate credit to the original author(s) and the source, provide a link to the Creative Commons license, and indicate if changes were made. Funded by SCOAP ${ }^{3}$.

\section{References}

1. L. Lukaszuk, B. Nicolescu, Lett. Nuovo Cim. 8, 405 (1973)

2. P. Gauron, B. Nicolescu, E. Leader, Phys. Lett. B 238, 406 (1990)

3. M. Fukugita, J. Kwiecinski, Phys. Lett. B 83, 119 (1979)

4. I.I. Balitsky, L.N. Lipatov, Sov. J. Nucl. Phys. 28, 822 (1978) [Yad. Fiz. 28, 1597 (1978)]

5. E.A. Kuraev, L.N. Lipatov, V.S. Fadin, Sov. Phys. JETP 45, 199 (1977) [Zh. Eksp. Teor. Fiz. 72, 377 (1977)]

6. E.A. Kuraev, L.N. Lipatov, V.S. Fadin, Sov. Phys. JETP 44, 443 (1976) [Zh. Eksp. Teor. Fiz. 71, 840 (1976)]

7. K. Akiba et al. (LHC Forward Physics Working Group), J. Phys. G 43, 110201 (2016). arXiv:1611.05079 [hep-ph]

8. J. Bartels, Nucl. Phys. B 175, 365 (1980)

9. J. Kwiecinski, M. Praszalowicz, Phys. Lett. B 94, 413 (1980)

10. T. Jaroszewicz, Acta Phys. Pol. B 11, 965 (1980)

11. L.N. Lipatov, JETP Lett. 59, 596 (1994). arXiv:hep-th/9311037 [Pisma Zh. Eksp. Teor. Fiz. 59, 571 (1994)]

12. L.D. Faddeev, G.P. Korchemsky, Phys. Lett. B 342, 311 (1995). arXiv:hep-th/9404173

13. H.J. De Vega, L.N. Lipatov, Phys. Rev. D 64, 114019 (2001). arXiv:hep-ph/0107225

14. H.J. De Vega, L.N. Lipatov, Phys. Rev. D 66, 074013 (2002). arXiv:hep-ph/0204245

15. L.N. Lipatov, AIP Conf. Proc. 1523, 247 (2012)

16. G. Chachamis, A. Sabio Vera, Phys. Rev. D 94(3), 034019 (2016). arXiv:1606.07349 [hep-ph]

17. R.A. Janik, J. Wosiek, Phys. Rev. Lett. 82, 1092 (1999). arXiv:hep-th/9802100

18. J. Bartels, L.N. Lipatov, G.P. Vacca, Phys. Lett. B 477, 178 (2000). arXiv:hep-ph/9912423

19. Y.V. Kovchegov, L. Szymanowski, S. Wallon, Phys. Lett. B 586, 267 (2004). arXiv:hep-ph/0309281

20. L.N. Lipatov, J. Phys. A 42, 304020 (2009). arXiv:0902.1444 [hepth]

21. J. Bartels, L.N. Lipatov, A. Sabio Vera, Phys. Rev. D 80, 045002 (2009). arXiv:0802.2065 [hep-th]
22. J. Bartels, L.N. Lipatov, A. Sabio Vera, Eur. Phys. J. C 65, 587 (2010). arXiv:0807.0894 [hep-th]

23. L.N. Lipatov, Theor. Math. Phys. 170, 166 (2012). arXiv:1008.1015 [hep-th]

24. G. Chachamis, A. Sabio Vera, Phys. Lett. B 709, 301 (2012). arXiv: 1112.4162 [hep-th]

25. G. Chachamis, A. Sabio Vera, Phys. Lett. B 717, 458 (2012). arXiv:1206.3140 [hep-th]

26. G. Chachamis, M. Deak, A. Sabio Vera, P. Stephens, Nucl. Phys. B 849, 28 (2011). arXiv:1102.1890 [hep-ph]

27. G. Chachamis, A. Sabio Vera, C. Salas, Phys. Rev. D 87(1), 016007 (2013). arXiv:1211.6332 [hep-ph]

28. F. Caporale, G. Chachamis, J.D. Madrigal, B. Murdaca, A. Sabio Vera, Phys. Lett. B 724, 127 (2013). arXiv:1305.1474 [hep-th]

29. G. Chachamis, A. Sabio Vera, Phys. Rev. D 93(7), 074004 (2016). arXiv:1511.03548 [hep-ph]

30. G. Chachamis, A. Sabio Vera, JHEP 1602, 064 (2016). arXiv:1512.03603 [hep-ph]

31. T. Bargheer, JHEP 1711, 077 (2017). arXiv:1606.07640 [hep-th]

32. J. Bondy, U. Murty, Graph Theory with Applications (Macmillan, Basingstoke, 1976)

33. R. Gurau, V. Rivasseau, A. Sfondrini, arXiv:1401.5003 [hep-th]

34. G. Kirchhoff, Über die Auflösung der Gleichungen, auf welche man bei der Untersuchung der linearen Verteilung galvanischer Ströme gefuhrt wird. Ann. Physik Chemie 72, 497-508 (1847)

35. J. Bartels, L.N. Lipatov, A. Sabio Vera, JHEP 1407, 056 (2014). arXiv:1208.3423 [hep-th]

36. A. Sabio Vera, M.A. Vazquez-Mozo, JHEP 1503, 070 (2015). arXiv:1412.3699 [hep-th]

37. H. Johansson, A. Sabio Vera, E. Serna Campillo, M.A. VazquezMozo, JHEP 1310, 215 (2013). arXiv:1307.3106 [hep-th]

38. A. Sabio Vera, E. Serna Campillo, M.A. Vazquez-Mozo, JHEP 1304, 086 (2013). arXiv:1212.5103 [hep-th]

39. J. Bartels, V. Schomerus, M. Sprenger, JHEP 1507, 098 (2015). arXiv:1411.2594 [hep-th]

40. J. Bartels, J. Kotanski, V. Schomerus, JHEP 1101, 096 (2011) arXiv: 1009.3938 [hep-th]

41. B. Basso, S. Caron-Huot, A. Sever, JHEP 1501, 027 (2015). arXiv:1407.3766 [hep-th]

42. R.C. Brower, M. Djuric, C.I. Tan, JHEP 0907, 063 (2009). arXiv:0812.0354 [hep-th]

43. A.V. Kotikov, L.N. Lipatov, Nucl. Phys. B 874, 889 (2013). arXiv:1301.0882 [hep-th]

44. M.S. Costa, V. Goncalves, J. Penedones, JHEP 1212, 091 (2012). arXiv:1209.4355 [hep-th]

45. M. Alfimov, N. Gromov, V. Kazakov, JHEP 1507, 164 (2015). arXiv: 1408.2530 [hep-th]

46. R.C. Brower, J. Polchinski, M.J. Strassler, C.I. Tan, JHEP 0712, 005 (2007). arXiv:hep-th/0603115

47. R.C. Brower, M.S. Costa, M. Djuric, T. Raben, C.I. Tan, JHEP 1502, 104 (2015). arXiv:1409.2730 [hep-th]

48. J. Bartels, V.S. Fadin, L.N. Lipatov, G.P. Vacca, Nucl. Phys. B 867, 827 (2013). arXiv:1210.0797 [hep-ph]

49. L.J. Dixon, J.M. Drummond, C. Duhr, J. Pennington, JHEP 1406, 116 (2014). arXiv:1402.3300 [hep-th]

50. S. Caron-Huot, L.J. Dixon, A. McLeod, M. von Hippel, Phys. Rev. Lett. 117(24), 241601 (2016). arXiv: 1609.00669 [hep-th]

51. L.J. Dixon, J. Drummond, T. Harrington, A.J. McLeod, G. Papathanasiou, M. Spradlin, JHEP 1702, 137 (2017). arXiv:1612.08976 [hep-th] 